\title{
Prognostic significance of age in patients with acute ischaemic stroke treated with intravenous thrombolysis
}

\author{
Marcin Wnuk $^{1,2}$, Leszek Drabik ${ }^{3,4}, J^{\prime}$ styna Derbisz ${ }^{1,2}$, Agnieszka Słowik $^{1,2}$ \\ ${ }^{1}$ Jagiellonian University Medical College, Department of Neurology, Krakow, Poland \\ ${ }^{2}$ Department of Neurology, University Hospital in Krakow, Poland \\ ${ }^{3}$ Jagiellonian University Medical College, Department of Pharmacology, Krakow, Poland \\ ${ }^{4}$ John Paul II Hospital, Krakow, Poland
}

\section{ABSTRACT}

Aim of the study. To assess the influence of age on long-term functional outcome in patients with acute ischaemic stroke (AIS) treated with intravenous thrombolysis (IVT).

Material and methods. We performed retrospective analysis of 362 AIS patients treated with IVT or IVT and subsequent mechanical thrombectomy in the University Hospital in Krakow, Poland. Patients were categorised into four subgroups by age: (I) below the age of 60, (II) 60 to 69, (III) 70 to 79, and (IV) 80 or more. The outcomes were assessed with modified Rankin scale (mRS) 90 days after stroke onset, and defined as favourable (mRS 0-2), poor (mRS 3-5), or death (mRS =6).

Results. Patients aged 80 or more compared to those below 60 were more often women (72.64\% vs. $26.76 \%$, $<0.001)$, more often suffered from hypertension $(94.34 \%$ vs. $60.56 \%, p<0.001)$, ischaemic heart disease $(27.36 \%$ vs. $8.45 \%$, $p=0.002)$, atrial fibrillation $(49.06 \%$ vs. $5.63 \%, p<0.001)$, and premorbid disability (pre-stroke $m R S \geq 1: 17.92 \%$ vs. $1.41 \%$, $p<0.001)$, less often were active smokers ( $0 \%$ vs. $27.14 \%, p<0.001)$, more often had cardioembolic aetiology $(50.00 \%$ vs. $16.90 \%, p<0.001)$, and less often other stroke aetiology (1.89\% vs. $15.49 \%,<0.008)$, had shorter time from stroke onset to IVT (125 [93-180] vs. 140 [110-186] min, $p<0.008)$, less often underwent mechanical thrombectomy $(18.87 \%$ vs. $46.48 \%$, $p<0.001$ ), had higher CRP levels (10.3 [3.2-39.8] vs. $4.3[2.1-9.6] \mathrm{mg} / \mathrm{L}, \mathrm{p}=0.003)$, higher maximal systolic blood pressure within 24 hours after IVT (153 [140-170] vs. 138 [120-145] mmHg, $p<0.001$ ), and higher creatinine concentration (88 [68-108] vs. 77 [67-87] $\mu \mathrm{mol} / \mathrm{l}, \mathrm{p}=0.004$ ), less often had a favourable outcome ( $48.04 \%$ vs. $85.51 \%$, odds ratio [OR] $0.16,95 \% \mathrm{Cl}: 0.07-0.34$, $\mathrm{p}<0.001)$, and had a greater risk of death $(26.47 \%$ vs. $5.80 \%$, OR $5.85,95 \%$ Cl: $1.95-17.59, \mathrm{p}<0.001)$ within three months of stroke onset. Multivariable logistic regression analysis showed that the independent predictors of worse outcome in patients aged 80 or more were NIHSS score after IVT (OR $0.64,95 \% \mathrm{Cl}: 0.53-0.78, p<0.001)$, pre-stroke mRS score $\geq 1$ (OR $0.10,95 \% \mathrm{Cl}$ : $0.02-0.61, p=0.012$ ), and CRP levels (OR 0.96, 95\%Cl: $0.93-0.99, p=0.007$ ).

Conclusions. AIS patients treated with reperfusion therapy and aged 80 or more have around a six times higher risk of an unfavourable outcome or death within three months of stroke onset compared to those aged below 60 . Higher NIHSS score after IVT, any signs of disability before stroke as measured with $\mathrm{mRS}$, and higher CRP levels are independent risk factors for worse prognosis in the elderly.

Key words: ischaemic stroke, intravenous thrombolysis, age, elderly, prognosis

(Neurol Neurochir Pol 2022; 56 (1): 81-87)

Address for correspondence: Marcin Wnuk, Jagiellonian University Medical College, Department of Neurology, Jakubowskiego 2 Str., 30-688 Krakow, Poland; e-mail: marcin.wnuk@uj.edu.pl

Received: 10.11.2021 Accepted: 20.12.2021 Early publication date: 21.01.2022

This article is available in open access under Creative Common Attribution-Non-Commercial-No Derivatives 4.0 International (CC BY-NC-ND 4.0) license, allowing to download articles and share them with others as long as they credit the authors and the publisher, but without permission to change them in any way or use them commercially. 


\section{Introduction}

Age is the most important non-modifiable risk factor for acute ischaemic stroke (AIS) [1]. Above the age of 45, stroke incidence doubles with each decade, and more than two-thirds of all cerebrovascular events occur after the age of 65 [2]. Due to the ageing population of Europe, increases in the incidence $(+3 \%)$ and prevalence $(+27 \%)$ of stroke are expected in the European Union over the next 30 years [3]. The COVID-19 pandemic additionally complicated the situation of patients with AIS, including the elderly, resulting in a significant decrease in the hospital admission rate and prolonged time to reperfusion therapies, especially during the first weeks of pandemic [4].

Data from a German stroke registry showed that the rate of treatment with intravenous thrombolysis (IVT) among patients with AIS decreased from 15\% in those aged below 50 to $8 \%$ among those aged 90 or more [5]. Previous older studies showed that patients with AIS treated with IVT and aged 80 or more had three times higher three-month mortality compared to those below 80 , with no significant difference in the rate of secondary symptomatic intracranial haemorrhage [6]. A recent Brazilian study revealed instead that, although patients with AIS treated with IVT or mechanical thrombectomy (MT) after the age of $80 \mathrm{had}$ an increased risk of an unfavourable outcome when compared to younger individuals, the mortality rate was similar in all studied age subgroups [7]. Previous older studies have highlighted that the prevalence of cerebrovascular risk factors such as hypertension, diabetes mellitus, atrial fibrillation, and dyslipidemia is higher in the elderly, possibly leading to increased mortality and unfavourable outcomes in cases of AIS treated with IVT [8].

Therefore, the aim of our study was to assess the influence of age categorised into four subgroups on long-term functional outcomes in patients with AIS treated with IVT in a sample of the Polish population.

\section{Material and methods}

We retrospectively analysed the data of 362 patients with AIS treated with IVT between June 2014 and December 2018. Patient data was collected prospectively in the University Hospital in Krakow as part of a single-centre registry known as the Krakow Stroke Data Bank. Our study focused on age further categorised into four subgroups in a similar way as in the previous study of the Brazilian stroke cohort [7], i.e.: (I) below 60, (II) 60 to 69, (III) 70 to 79, and (IV) 80 or above.

The data on cardiovascular risk factors and outcome measures was collected as described previously [9]. In brief, we obtained information regarding demographics, pre-stroke modified Rankin scale (mRS), presence of hypertension, ischaemic heart disease, atrial fibrillation, diabetes mellitus, hypercholesterolemia, smoking habits, AIS aetiology, time from stroke onset to IVT, and National Institutes of Health
Stroke Scale (NIHSS) recorded on admission and after IVT. $\mathrm{C}$-reactive protein (CRP) levels were measured during the first 72 hours after hospital admission. Fasting glucose was measured in serum the next morning after IVT, as described previously [9]. The outcome was assessed with mRS 90 days after stroke onset, and was defined as favourable (mRS 0-2), poor (mRS 3-5), or death $(m R S=6)$. Secondary haemorrhagic brain complications caused by IVT were defined in accordance with the classification of the European Cooperative Acute Stroke Study (ECASS-1) [10].

This study received the approval of the Jagiellonian University Ethical Committee (KBET54/B/2007), and was conducted in accordance with the Declaration of Helsinki. Informed consent, either written or - in case of inability to use the dominant hand due to AIS - verbal in the presence of at least two physicians, was collected from each patient participating in the study.

The baseline clinical characteristics according to the age categories were presented as counts and percentages, median and interquartile range. The continuous data was tested for normality of distribution with a Shapiro-Wilk test. A Kruskal-Wallis test and test for multiple comparisons of mean rank were used to compare medians. Categorical variables were compared with the chi-square test and Fisher's exact test, and for pairwise comparisons, multiple hypothesis correction was performed using the Bonferroni method.

We performed logistic regression analysis to identify independent predictors of the 90-day outcome after AIS according to the age category. Multivariable models were built using backward stepwise elimination based on Akaike information criterion (AIC) levels. Models were adjusted for sex, NIHSS score, haemorrhagic brain complications after IVT, and CRP levels. The area under the receiver operating characteristic curve (AUC) with 95\% confidence intervals (CI) was calculated to show the discriminative ability of the prediction models. All statistical tests were 2-sided, and a p-value of 0.05 was considered statistically significant. Data was analysed using STATISTICA version 13 (Statsoft Inc, Tulsa, OK, USA).

The data that confirms the results of the current study is available from the corresponding author upon reasonable request.

\section{Results}

\section{Patient characteristics}

Our study included 362 patients with AIS treated with IVT. One hundred and eight patients (29.8\%) underwent also mechanical thrombectomy (MT). The prevalence of hypertension, atrial fibrillation, non-smoking, cardioembolic stroke aetiology and premorbid disability increased with each age category (Tab. 1). With increasing age categories, the chance of a favourable outcome gradually decreased, and the risk of death increased (Fig. 1). 
Table 1. Baseline characteristics of patients according to age categories

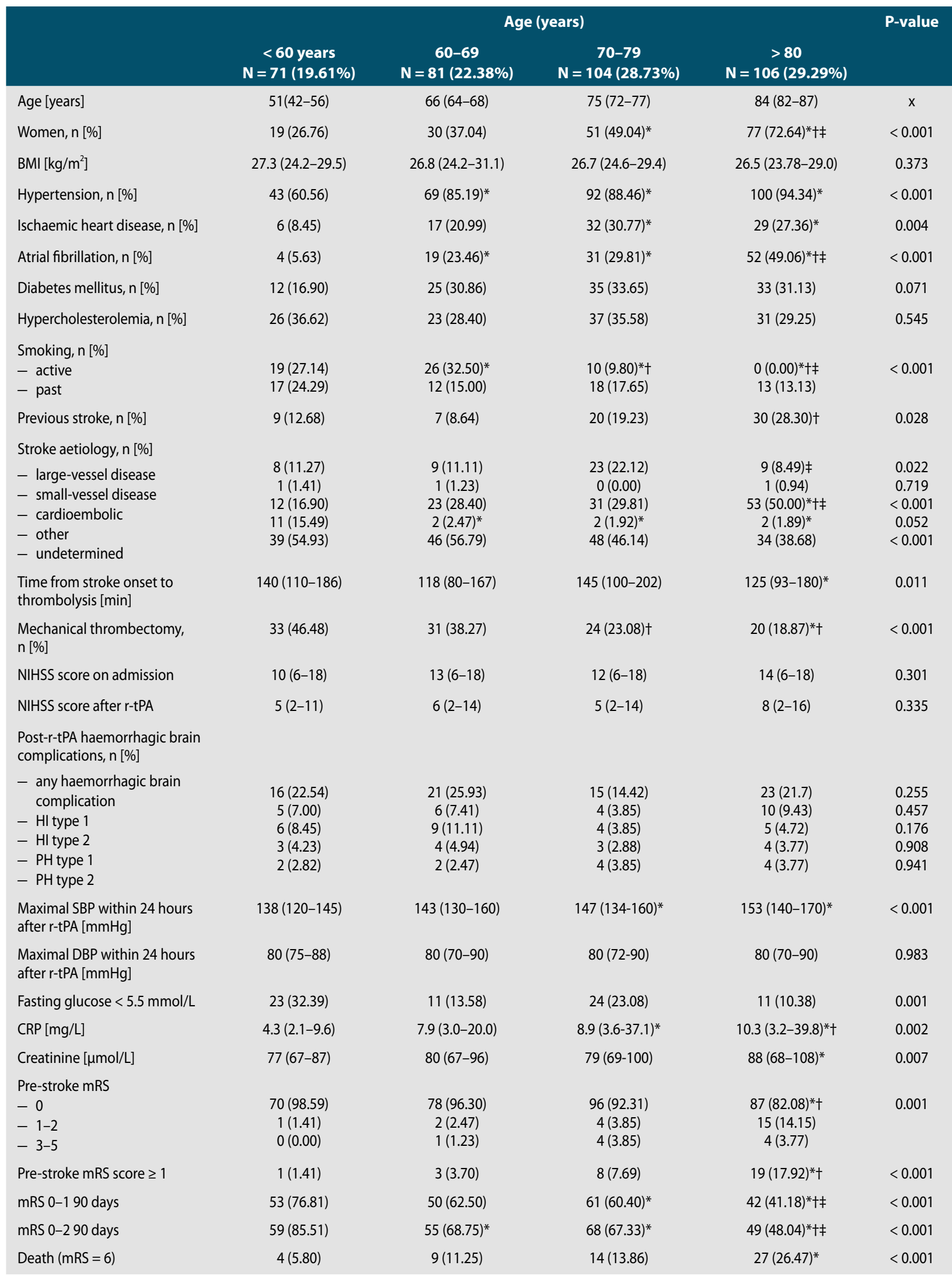

Bonferroni adjusted $p$-values: ${ }^{*} P<0.008$, vs. $<60$ years; $\uparrow P<0.008$, vs. 60-69 years; $¥ \mathrm{P}<0.008$, vs. $70-79$ years. Values are presented as $n(\%)$, median and interquartile range. $B M I-$ body mass index; $C R P$ - C-reactive protein; DBP — diastolic blood pressure; $\mathrm{HI}$ - haemorrhagic infarction; mRS - modified Rankin scale; MT — mechanical thrombectomy; NIHSS - National Institutes of Health Stroke Scale; PH parenchymal haematoma; r-tPA — recombinant tissue plasminogen activator; SBP — systolic blood pressure 


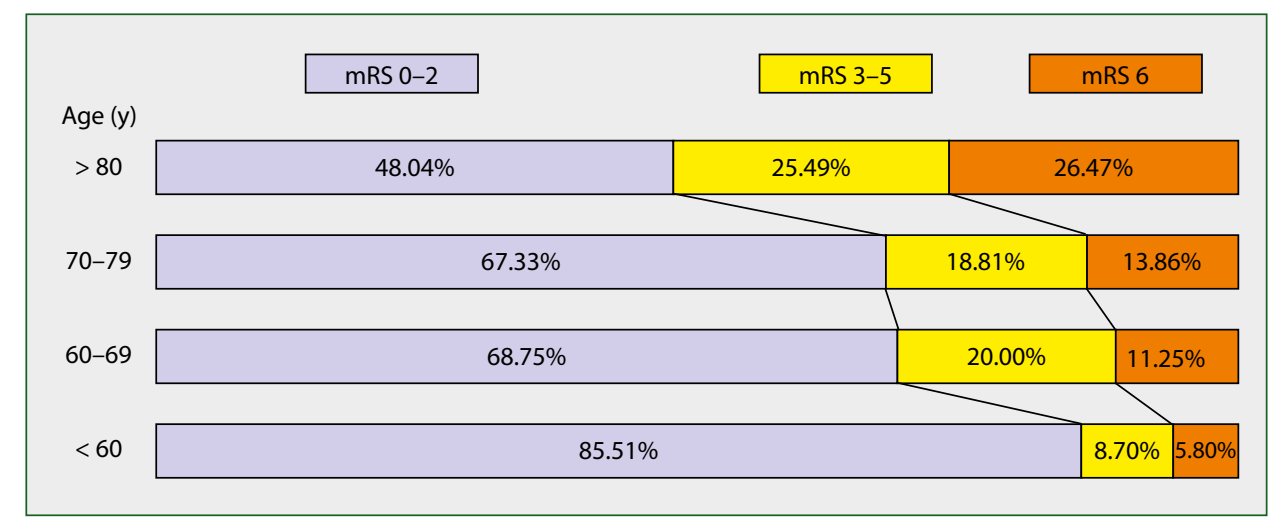

Figure 1. Proportions of patients with favourable outcome (modified Rankin scale [mRS] 0-2), poor outcome (mRS 3-5), and those who died $(\mathrm{mRS}=6)$ three months after stroke onset, according to age categories

\section{Comparison of age subgroups}

Patients aged 80 or more $(n=106$, median age 84 [82-87]) compared to those below $60(n=71$, median age 51 [42-56]) were more often women, more often suffered from hypertension, ischaemic heart disease, atrial fibrillation and pre-stroke disability, less often were active smokers, more often had cardioembolic and less often other stroke aetiology, had shorter time from stroke onset to IVT, less often underwent MT, had higher CRP levels, higher creatinine concentration, and higher maximal systolic blood pressure within 24 hours after IVT, less often had favourable outcome ( $48.04 \%$ vs. $85.51 \%, \mathrm{p}<0.001)$, and had higher mortality 90 days after stroke onset $(26.47 \%$ vs. $5.80 \%, \mathrm{p}<0.001$ ) (Tab. 1, Fig. 1). Patients aged 80 or more had a more than six times higher risk of achieving mRS 3-690 days after stroke onset compared to those aged below 60 (OR 6.38, 95\%CI: 2.94-13.84, $\mathrm{p}<0.001$ ) (Fig. 2).

\section{Determinants of favourable outcome}

Patients aged 80 or more who had mRS 3-6 90 days after stroke onset $(n=53)$ compared to those with favourable outcome $(\mathrm{n}=49)$ had higher NIHSS score on admission $(16$ [14-20] vs. 7 [5-12], p < 0.001) and after IVT (15 [8-18] vs. 2 [1-6], $\mathrm{p}<0.001)$, more often had haemorrhagic brain complications after IVT ( $35.85 \%$ vs. $8.16 \%, \mathrm{p}<0.001)$ and premorbid disability (pre-stroke $\mathrm{mRS}$ score $\geq 1: 28.30 \%$ vs. $8.16 \%, \mathrm{p}=0.010$ ), less often had fasting glucose level below $5.5 \mathrm{mmol} / \mathrm{L}(9.43 \%$ vs. $26.53 \%, \mathrm{p}=0.023)$, had higher CRP levels (27.2 [9.1-84.1] vs. 4.3 [2.1-10.3] mg/L, p < 0.001), and had higher maximal diastolic blood pressure within 24 hours after IVT (80 [72-90] vs. 80 [70-85] mmHg, $\mathrm{p}=0.026$ ) (Supplemental Tab. 1). Patients below 60 who had mRS 3-6 90 days after stroke onset $(n=10)$ compared to those with a favourable outcome $(n=59)$ more often suffered from hypertension ( $90.00 \%$ vs. $54.24 \%, \mathrm{p}=0.033$ ), more often underwent MT ( $90.00 \%$ vs. $38.98 \%, p=0.028)$, had higher NIHSS score on admission (19 [18-20] vs. 9 [5-16], p = $0.005)$ and after IVT (19 [15-19] vs. 4 [2-8], p < 0.001), more often had haemorrhagic brain complications after IVT $(70.00 \%$

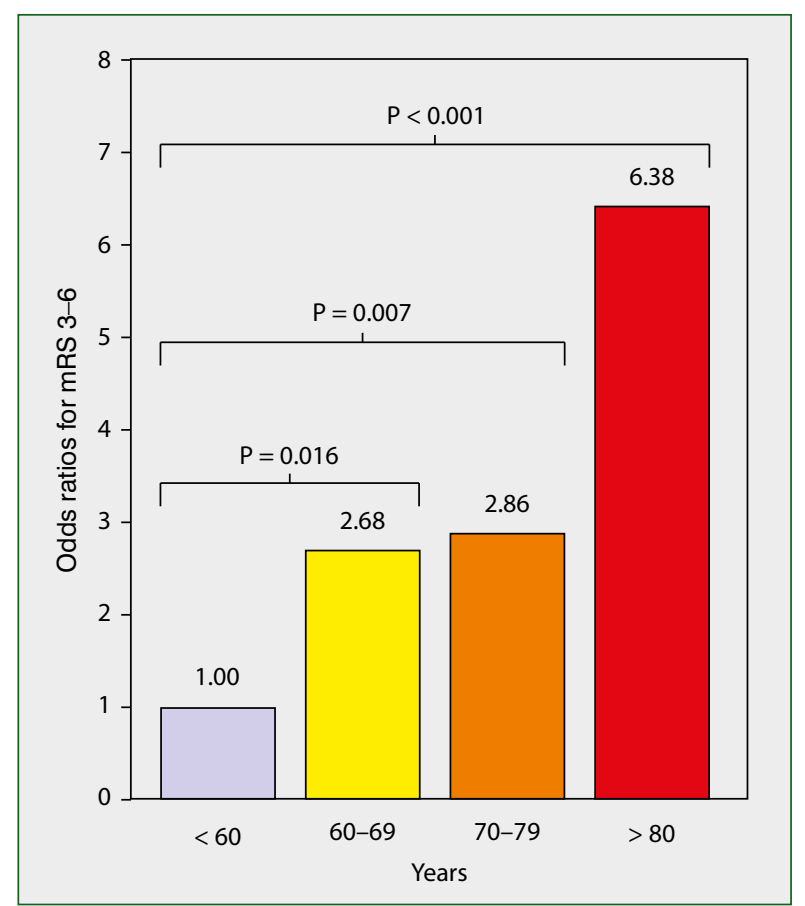

Figure 2. Odds ratios for modified Rankin scale 3-6 three months after stroke onset in studied age categories

vs. $14.56 \%, \mathrm{p}<0.001)$, and had higher CRP levels (9.3 [7.2-14.0] vs. $4.0[1.5-7.7] \mathrm{mg} / \mathrm{L}, \mathrm{p}=0.018$ ) (Supplemental Tab. 1).

More than two-thirds of patients with a pre-stroke mRS score of 0 experienced a favourable outcome 90 days after stroke onset, whereas $13 \%$ of patients without premorbid disability died during long-term observation (Supplemental Tab. 2). There were only nine patients with a pre-stroke mRS score of 3-5, and two of them died within 90 days of stroke onset (Supplemental Tab. 2).

Multivariable logistic regression analysis revealed that independent predictors of a favourable outcome were: lower 
Table 2. Multivariable regression analysis for mRS 0-2 according to age categories

\begin{tabular}{|c|c|c|c|c|c|c|c|c|}
\hline & \multicolumn{2}{|c|}{$<60$ years } & \multicolumn{2}{|c|}{$60-69$ years } & \multicolumn{2}{|c|}{$70-79$ years } & \multicolumn{2}{|c|}{$>80$ years } \\
\hline & OR $(95 \% \mathrm{CI})$ & P-value & OR (95\% CI) & P-value & OR $(95 \% \mathrm{Cl})$ & P-value & OR $(95 \% \mathrm{CI})$ & P-value \\
\hline Women & - & - & $0.17(0.04-0.82)$ & 0.027 & - & - & - & - \\
\hline Pre-stroke $\mathrm{mRS}$ score $\geq 1$ & - & - & - & - & $0.06(0.01-0.58)$ & 0.015 & $0.10(0.02-0.61)$ & 0.012 \\
\hline $\begin{array}{l}\text { NIHSS score after r-tPA } \\
\text { (per point) }\end{array}$ & $0.68(0.56-0.84)$ & $<0.001$ & $0.73(0.63-0.84)$ & $<0.001$ & $0.68(0.58-0.79)$ & $<0.001$ & $0.64(0.53-0.78)$ & $<0.001$ \\
\hline $\begin{array}{l}\text { Post-r-tPA haemorrhagic } \\
\text { brain complications }\end{array}$ & - & - & - & - & - & - & - & - \\
\hline $\mathrm{CRP}[\mathrm{mg} / \mathrm{L}]$ & - & - & - & - & - & - & $0.96(0.93-0.99)$ & 0.007 \\
\hline AIC & 32.91 & & 52.91 & & 54.89 & & 63.67 & \\
\hline $\operatorname{AUC}(95 \% \mathrm{Cl})$ & $0.93(0.87-0.99)$ & & $0.94(0.89-0.99)$ & & $0.95(0.91-0.99)$ & & $0.96(0.93-0.99)$ & \\
\hline Hosmer-Lemeshow test & 0.152 & & 0.945 & & 0.027 & & 0.410 & \\
\hline
\end{tabular}

$\mathrm{AIC}$ - Akaike information criterion; AUC — area under receiver operating characteristic curve, $\mathrm{Cl}$ - confidence intervals; $\mathrm{OR}$ - odds ratio

NIHSS score after IVT in all age subgroups, male sex in patients aged between 60 and 69, the lack of pre-stroke disability in patients aged 70 or more, and lower CRP levels in patients aged 80 or more (Tab. 2).

\section{Patients treated with IVT and subsequent MT}

Additional analyses were performed in the subgroup of patients restricted to those treated with IVT and subsequent MT. Patients aged 80 or more $(n=20$, median age 83 [81-86]) compared to those below $60(n=33$, median age 51 [42-55]) were more often women $(90.00 \%$ vs. $21.21 \%, \mathrm{p}<0.001)$, more often suffered from hypertension $(100.00 \%$ vs. $63.64 \%, \mathrm{p}=$ $0.021)$, more often had cardioembolic aetiology $(55.00 \%$ vs. $18.18 \%, \mathrm{p}<0.001)$, had shorter time from stroke onset to IVT (102 [73-129] vs. 143 [114-145] minutes, $p=0.012)$, and less often had a favourable outcome ( $31.58 \%$ vs. $71.88 \%, p=0.012)$ (Supplemental Tab. 3, Supplemental Figure 1). In contrast to the whole group, there were no significant differences in patients aged 80 or more compared to those below 60 regarding CRP levels (11.4 [5.7-26.2] vs. 7.2 [2.6-11.0] mg/L, $\mathrm{p}=0.237$ ) or mortality 90 days after stroke onset $(31.58 \%$ vs. $9.38 \%, \mathrm{p}=$ 0.260 ). Patients aged 80 or more had a more than five times higher risk of achieving mRS 3-6 90 days after stroke onset compared to those below 60 (OR $=5.53,95 \% \mathrm{CI}$ : 1.61-19.07, $\mathrm{p}=0.008)$ (Supplemental Figure 2).

Patients aged 80 or more who had mRS 3-690 days after stroke onset $(\mathrm{n}=13)$ compared to those with a favourable outcome $(n=6)$ had a higher NIHSS score after IVT $(15$ [8-19] vs. $4[2-10], p=0.018$ ) (Supplemental Tab. 4). However, there were no significant differences between the subgroups according to premorbid disability (pre-stroke mRS score $\geq 1$ : $30.77 \%$ vs. $0.00 \%, \mathrm{p}=0.255)$ or CRP levels (15.4 [7.1-27.4] vs. 10.1 [4.2-25.1] mg/L, $\mathrm{p}=0.511$ ) (Supplemental Tab. 4). Patients below 60 who had mRS 3-6 90 days after stroke onset $(n=9)$ compared to those with a favourable outcome (n = 23) had higher NIHSS score on admission (19 [18-20] vs. 16 [10-18], p = 0.015) and after IVT (19 [16-19] vs. 6 [3-11], $\mathrm{p}<0.001)$ and more often had haemorrhagic brain complications after IVT (77.78\% vs. 26.09\%, p = 0.031) (Supplemental Tab. 4). Multivariable logistic regression analysis was not performed due to the small number of patients in the analysed subgroups.

\section{Discussion}

In our study, for the first time in a sample of Polish AIS patients we have evaluated stroke reperfusion therapy in four age categories: (I) below 60, (II) 60 to 69 , (III) 70 to 79 , and (IV) 80 or more. We were able to show that patients with AIS treated with IVT and aged 80 or more had a more than six times lower chance of a favourable outcome and a nearly six times greater risk of death within three months of stroke onset compared to those below 60 . Our findings accord with a recent study performed on more than 500 rural residents of Poland that also revealed greater mortality and higher rate of functional dependence in thrombolysed patients aged 80 or more years compared to the remainder [11]. Similar conclusions came from a study of a Hungarian cohort comprising more than 1,000 AIS patients treated with IVT: this also showed a significantly lower chance of 1-year survival in the elderly [12]. Data coming from a previous systematic review of six studies including more than 2,000 AIS patients, among whom nearly one in four were aged 80 or more, also supported the results of our study [6]. In contrast, a recent study of a Brazilian cohort where patients treated with IVT and MT were analysed together showed that those aged 80 or more did not exhibit greater mortality, although they had less favourable outcomes at discharge and at three months after stroke onset [7].

Our study also revealed that elderly patients with AIS treated with IVT significantly more often were women, suffered from atrial fibrillation (AF), and had cardioembolic stroke aetiology. Our findings align with previous Hungarian research in which patients after the age of 80 had AF nearly three times as often as the remainder [12]. It is known that cardioembolic 
stroke, most commonly caused by AF [13], is associated with increased long-term mortality compared to other aetiologies, even after adjustments for age and stroke severity [14]. Pooled analysis of more than 6,000 individuals with AIS showed also that women presented with more severe strokes. Although after adjustments for age and AF this association was attenuated, it still remained statistically significant [15]. Similarly, in a cohort of 1,830 Polish AIS patients treated with alteplase, women also exhibited worse long-term outcomes compared to men, probably due to their older age and higher frequency of cardioembolic stroke presenting with more severe neurological deficit [16].

When patients were analysed according to the mRS, it occurred that in all age subgroups worse outcome was associated with bleeding brain complications, CRP levels and NIHSS score, both on admission and after IVT. However, the multivariable model showed that NIHSS score after IVT was the only independent risk factor for worse outcome across all age subgroups. To date, studies have concentrated mainly on NIHSS score on admission $[17,18]$. In a recent Italian study of nearly 500 AIS patients, higher NIHSS score was associated with an unfavourable outcome and the risk of death independently from treatment with IVT [19].

In the present study in patients aged 80 or more, higher CRP levels additionally were independently associated with worse functional outcome. As shown in our previous study, CRP levels evaluated in a different time window, i.e. 1224 hours after IVT, were an independent risk factor for poor short-term and long-term outcomes and were also associated with in-hospital mortality [20]. Analysis of healthy individuals showed that people aged 65 or more had significantly higher CRP levels than their younger counterparts [21]. Moreover, CRP elevations in cases of stroke might reflect its severity [22]. Therefore, higher CRP levels, especially in the elderly, might play an important prognostic role in AIS treated with IVT. Previous studies have also suggested other potential risk factors for worse outcome of AIS in the elderly, such as antiplatelet or anticoagulant use before stroke, and higher white blood cell count [11]. A Chinese study showed that in older individuals with AIS, thrombolysis led to greater benefit in patients with lower NIHSS at baseline or those with posterior circulation infarct [23]. On the other hand, haemorrhagic brain complications were not found to be an independent risk factor for worse outcome in our study. Previous studies showed that the rate of intracerebral haemorrhage secondary to IVT, even when symptomatic, was similar in patients below and above the age of 80 [12]. Treatment with IVT significantly increased the number of patients with favourable outcome, defined as mRS of $0-2$ at discharge in all age groups, including the elderly [5]. Even in patients after the age of 100, IVT could be efficiently used with no increase in the risk of secondary bleeding brain complications [24]. The same applied to those aged 90 or more years in the context of another type of reperfusion therapy, i.e. endovascular therapy, which did not increase the risk of symptomatic intracranial haemorrhage either [25].
Our study showed that premorbid disability, defined as pre-stroke mRS score of 1 point or more, was an independent risk factor for worse outcome in patients treated with IVT and aged 70 or more. Previous analysis of a large database from the Safe Implementation of Treatments in Stroke - Eastern Europe (SITS-EAST) registry revealed that a pre-stroke mRS score of 1 point or more was associated with an increased risk of death and a lower chance of favourable outcome within three months of a stroke treated with IVT [26]. Similar conclusions came from prospectively collected data from four major stroke centres in the United Kingdom [27]. Moreover, patients with premorbid disability were significantly older and had higher NIHSS score before IVT than the remainder $[26,28,29]$. Patients with premorbid disability were also less likely to improve within 24 hours after IVT [28]. On the other hand, a recent Italian study found that patients with pre-stroke disability, defined as mRS score of 3 or 4 treated with IVT, did not exhibit greater 3-month mortality compared to those who did not receive IVT even though thrombolysed patients were significantly older [30]. Another study showed that the benefit from thrombolysis depends rather on the patient's sex, and that this association is independent of pre-stroke mobility impairment [31].

When our analysis was restricted only to patients treated with IVT and subsequent MT, it occurred that in contrast to the whole group no significant differences were found between patients aged 80 or more and those below 60 regarding CRP levels and mortality 90 days after stroke onset. As the number of subjects included in this analysis is low, further studies are needed to evaluate whether other factors, such as NIHSS score after IVT, have a higher prognostic value. A previous Japanese study of patients who underwent MT showed that older age was not an independent risk factor for worse outcome, in contrast to NIHSS score and a modified Treatment in Cerebral Ischaemia (mTICI) score of 3 consistent with complete reperfusion [32]. Moreover, in patients aged 80 or more, the only predictive factor for worse outcome was an mTICI score of 3 [32]. A recent analysis of the 'MR CLEAN' trial (Multicentre Randomised Clinical Trial of Endovascular Treatment for Acute Ischaemic Stroke) revealed that patients aged 80 or more had worse functional outcome and increased mortality than younger patients, although older patients had a higher relative benefit of successful reperfusion [33].

Our study has some potential limitations. Firstly, the cohort of our AIS patients treated with IVT was rather small, although the median age value was distributed proportionally within the subgroups. Secondly, one-third of our patients underwent additional MT that could influence the results of our study, as elderly patients were less often qualified to this procedure and besides NIHSS score on admission did not differ from their younger counterparts. However, we performed additional analyses restricted to patients treated with IVT and subsequent MT. Finally, the results of the multivariable analysis need to be interpreted with caution, because due to 
the small sample size only a few of the potential risk factors could be incorporated [34].

\section{Conclusions}

Our study demonstrates that AIS patients treated with IVT and aged 80 or more have about 6 times higher risk of unfavourable outcome or death three months after stroke onset compared to those aged below 60 . Higher NIHSS score after IVT is an independent risk factor for worse prognosis in all age subgroups, whereas the prognostic significance of premorbid disability and of higher CRP levels is restricted to patients older than 70 and 80 years, respectively.

Conflict of interest: None.

Funding: None.

\section{References}

1. Yousufuddin $\mathrm{M}$, Young $\mathrm{N}$, Yousufuddin $\mathrm{M}$, et al. Impact of multiple chronic conditions in patients hospitalized with stroke and transient ischemic attack. J Stroke Cerebrovasc Dis. 2017; 26(6): 12391248, doi: 10.1016/j.jstrokecerebrovasdis.2017.01.015, indexed in Pubmed: 28285088.

2. Kelly-Hayes M. Influence of age and health behaviors on stroke risk: lessons from longitudinal studies. J Am Geriatr Soc. 2010; 58 Suppl 2: S325-S328, doi: 10.1111/j.1532-5415.2010.02915.x, indexed in Pubmed: 21029062.

3. Wafa HA, Wolfe CDA, Emmett E, et al. Burden of stroke in Europe: thirty-year projections of incidence, prevalence, deaths, and disability-adjusted life years. Stroke. 2020; 51(8): 2418-2427, doi: 10.1161/ STROKEAHA.120.029606, indexed in Pubmed: 32646325.

4. Dębiec A, Bilik M, Piasecki P, et al. Effect of COVID-19 pandemic on stroke admissions and quality of stroke interventional treatment in Masovian Voivodeship. Neurol Neurochir Pol. 2021; 55(2): 223-226, doi: 10.5603/PJNNS.a2021.0025, indexed in Pubmed: 33783813.

5. Reuter B, Gumbinger C, Sauer T, et al. Stroke Working Group of Baden-Wuerttemberg, Stroke Working Group of Baden-Wuerttemberg. Intravenous thrombolysis for acute ischaemic stroke in the elderly: data from the Baden-Wuerttemberg stroke registry. Eur J Neurol. 2016; 23(1): 13-20, doi: 10.1111/ene.12829, indexed in Pubmed: 26492944.

6. Engelter ST, Bonati LH, Lyrer PA. Intravenous thrombolysis in stroke patients of $>$ or $=80$ versus $<80$ years of age--a systematic review across cohort studies. Age Ageing. 2006; 35(6): 572-580, doi: 10.1093/ageing/afl104, indexed in Pubmed: 17047006.

7. Furlan NE, Luvizutto GJ, Hamamoto Filho PT, et al. The impact of age on mortality and disability in patients with ischemic stroke who underwent cerebral reperfusion therapy: a Brazilian cohort study. Front Aging Neurosci. 2021; 13: 649902, doi: 10.3389/fnagi.2021.649902, indexed in Pubmed: 34295238.

8. Asdaghi N, Butcher KS, Hill MD. Risks and benefits of thrombolysis in the elderly. Int J Stroke. 2012; 7(2): 142-149, doi: 10.1111/j.1747-4949.2011.00744.x, indexed in Pubmed: 22264367.

9. Wnuk M, Derbisz J, Drabik L, et al. Fasting normoglycemia after intravenous thrombolysis predicts favorable long-term outcome in non-diabetic patients with acute ischemic stroke. J Clin Med. 2021; 10(14), doi: 10.3390/jcm10143005, indexed in Pubmed: 34300171.

10. Trouillas $P$, von Kummer R. Classification and pathogenesis of cerebral hemorrhages after thrombolysis in ischemic stroke. Stroke. 2006; 37(2): 556-561, doi: 10.1161/01.STR.0000196942.84707.71, indexed in Pubmed: 16397182.

11. Sobolewski P, Brola W, Wilczyński J, et al. Cerebral Thrombolysis in Rural Residents Aged $\geq 80$. Clinical Interventions in Aging. 2020; Volume 15: 1737-1751, doi: 10.2147/cia.s256070.

12. Héja $M$, Fekete I, Horváth $L$, et al. Experiences with intravenous thrombolysis in acute ischemic stroke by elderly patients-a "real world scenario". Front Neurol. 2021; 12: 721337, doi: 10.3389/ fneur.2021.721337, indexed in Pubmed: 34589048.

13. Khurshid S, Trinquart L, Weng LC, et al. Atrial fibrillation risk and discrimination of cardioembolic from noncardioembolic stroke. Stroke. 2020; 51(5): 1396-1403, doi: 10.1161/STROKEAHA.120.028837, indexed in Pubmed: 32252601.

14. Stead LG, Gilmore RM, Bellolio MF, et al. Cardioembolic but not other stroke subtypes predict mortality independent of stroke severity at presentation. Stroke Res Treat. 2011; 2011: 281496, doi: 10.4061/2011/281496, indexed in Pubmed: 22007347.

15. Phan HT, Reeves MJ, Blizzard CL, et al. Sex differences in long-term mortality after stroke in the INSTRUCT (INternational STRoke oUtComes sTudy): a meta-analysis of individual participant data. Circ Cardiovasc Qual Outcomes. 2017; 10(2): e010235, doi: 10.1161/CIRCOUTCOMES.116.003436, indexed in Pubmed: 28228454.

16. Wiszniewska M, Fryze W, Wiśniewska A, et al. Sex-related differences among ischaemic stroke patients treated with intravenous thrombolysis in Poland. Neurol Neurochir Pol. 2020; 54(3): 272-276, doi: 10.5603/PJNNS.a2020.0040, indexed in Pubmed: 32469076.

17. Amitrano D, Silva IR, Liberato BB, et al. Simple prediction model for unfavorable outcome in ischemic stroke after intravenous thrombolytic therapy. Arq Neuropsiquiatr. 2016; 74(12): 986-989, doi: 10.1590/0004-282X20160152, indexed in Pubmed: 27991996.

18. Chung $\mathrm{CC}$, Hong $\mathrm{CT}$, Huang $\mathrm{YH}$, et al. Predicting major neurologic improvement and long-term outcome after thrombolysis using artificial neural networks. J Neurol Sci. 2020; 410: 116667, doi: 10.1016/j. jns.2020.116667, indexed in Pubmed: 31978861.

19. Bandettini di Poggio M, Finocchi C, Brizzo F, et al. Management of acute ischemic stroke, thrombolysis rate, and predictors of clinical outcome. Neurol Sci. 2019; 40(2): 319-326, doi: 10.1007/s10072018-3644-3, indexed in Pubmed: 30430315.

20. Wnuk M, Derbisz J, Drabik L, et al. C-reactive protein and white blood cell count in non-infective acute ischemic stroke patients treated with intravenous thrombolysis. J Clin Med. 2021; 10(8), doi: 10.3390/ jcm10081610, indexed in Pubmed: 33920119.

21. Wyczalkowska-Tomasik A, Czarkowska-Paczek B, Zielenkiewicz M, et al. Inflammatory markers change with age, but do not fall beyond reported normal ranges. Arch Immunol Ther Exp (Warsz). 2016; 64(3): 249-254, doi: 10.1007/s00005-015-0357-7, indexed in Pubmed: 26283530.

22. Bustamante A, Simats A, Vilar-Bergua A, et al. Blood/brain biomarkers of inflammation after stroke and their association with outcome: from C-reactive protein to damage-associated molecular patterns. Neurotherapeutics. 2016; 13(4): 671-684, doi: 10.1007/s13311016-0470-2, indexed in Pubmed: 27538777.

23. Xu M, Guo J, Tao X, et al. The efficacy and safety of intravenous thrombolysis in older Chinese patients with acute ischemic stroke. 
Neurol India. 2021; 69(1): 91-96, doi: 10.4103/0028-3886.310086, indexed in Pubmed: 33642277.

24. Baena Álvarez B, García-Madrona S, Sainz Amo R, et al. Intravenous thrombolysis for acute ischemic stroke in centenarians. Eur Geriatr Med. 2021; 12(4): 893-897, doi: 10.1007/s41999-021-00494-4, indexed in Pubmed: 33909269.

25. Fujita K, Tanaka K, Yamagami $\mathrm{H}$, et al. Outcomes of large vessel occlusion stroke in patients aged $\geq 90$ years. Stroke. 2021; 52(5): 1561-1569, doi: 10.1161/STROKEAHA.120.031386, indexed in Pubmed: 33641385.

26. Karlinski M, Kobayashi A, Czlonkowska A, et al. Safe Implementation of Treatments in Stroke-Eastern Europe (SITS-EAST) Investigators. Role of preexisting disability in patients treated with intravenous thrombolysis for ischemic stroke. Stroke. 2014; 45(3): 770-775, doi: 10.1161/STROKEAHA.113.003744, indexed in Pubmed: 24496395.

27. Han TS, Fry CH, Gulli G, et al. Prestroke disability predicts adverse poststroke outcome: a pegistry-based prospective cohort study of acute stroke. Stroke. 2020; 51(2): 594-600, doi: 10.1161/STROKEAHA.119.027740, indexed in Pubmed: 31842700.

28. Cooray C, Karlinski M, Kobayashi A, et al. Safety and early outcomes after intravenous thrombolysis in acute ischemic stroke patients with prestroke disability. Int J Stroke. 2021; 16(6): 710-718, doi: 10.1177/1747493020954605, indexed in Pubmed: 32878588.

29. Zhang W, Coote S, Frost T, et al. Acute stroke patients with mild-to-moderate pre-existing disability should be considered for thrombolysis treatment. J Stroke Cerebrovasc Dis. 2018; 27(10): 2707-2711, doi: 10.1016/j.jstrokecerebrovasdis.2018.05.051, indexed in Pubmed: 30037650.

30. Merlino G, Corazza E, Lorenzut S, et al. Efficacy and safety of intravenous thrombolysis in patients with acute ischemic stroke and pre $\square$ existing disability. J Clin Med. 2019; 8(3), doi: 10.3390/jcm8030400, indexed in Pubmed: 30909477.

31. Lee SJ, Heo SH, Ambrosius WT, et al. Factors mediating outcome after stroke: gender, thrombolysis, and their interaction. TransI Stroke Res. 2018; 9(3): 267-273, doi: 10.1007/s12975-017-0579-6, indexed in Pubmed: 29067622.

32. Imahori T, Tanaka K, Arai A, et al. Mechanical thrombectomy using the Trevo ProVue in 50 consecutive patients with anterior circulation stroke: a single-center experience after approval of the stent retriever in Japan. Neurol Med Chir (Tokyo). 2017; 57(3): 128-135, doi: 10.2176/ nmc.0a.2016-0215, indexed in Pubmed: 28025467.

33. Groot AE, Treurniet KM, Jansen IGH, et al. MR CLEAN Registry Investigators. Endovascular treatment in older adults with acute ischemic stroke in the MR CLEAN Registry. Neurology. 2020; 95(2): e131e139, doi: 10.1212/WNL.0000000000009764, indexed in Pubmed: 32527972.

34. Wnuk M, Sawczyńska K, Kęsek T, et al. Neurological symptoms in hospitalised patients with COVID-19 and their association with in-hospital mortality. Neurol Neurochir Pol. 2021; 55(3): 314-321, doi: 10.5603/PJNNS.a2021.0039, indexed in Pubmed: 34037979. 